Article

\title{
Discrete Integrals and Axiomatically Defined Functionals
}

\author{
Erich Peter Klement ${ }^{1, *}$ and Radko Mesiar ${ }^{2}$
}

${ }^{1}$ Department of Knowledge-Based Mathematical Systems, Johannes Kepler University, Altenbergerstraße 69, Linz, Austria

2 Department of Mathematics and Descriptive Geometry, Faculty of Civil Engineering, Slovak University of Technology, Radlinského 11, Bratislava, Slovakia; E-Mail: mesiar@math.sk

* Author to whom correspondence should be addressed; E-Mail: ep.klement@jku.at; Tel.: +43-732-2468-4141; Fax: +43-732-2468-4142.

Received: 5 March 2012; in revised form: 13 April 2012 / Accepted: 13 April 2012 /

Published: 20 April 2012

\begin{abstract}
Several discrete universal integrals on finite universes are discussed from an axiomatic point of view. We start from the first attempt due to B. Riemann and cover also most recent approaches based on level dependent capacities. Our survey includes, among others, the Choquet and the Sugeno integral and general copula-based integrals.
\end{abstract}

Keywords: capacity; Choquet integral; copula; fuzzy integral; OWA operator; OMA operator; Sugeno integral

\section{Introduction}

Constructive approaches to integration can be traced as far back as ancient Egypt around 1850 BC: the Moscow Mathematical Papyrus (Problem 14) contains a formula for the volume of a frustum of a square pyramid. The first documented systematic technique capable of determining integrals is the method of exhaustion of the ancient Greek astronomer EuDOXUS (ca. 370 BC) who tried to find areas and volumes by approximating them by a (large) number of shapes for which the area or volume was known. This method was further developed by ARCHIMEDES in the third century BC who calculated the area of parabolas and gave an approximation to the area of a circle. Similar methods were independently developed in China around the third century AD by LIU HUI, who used it to find the area of the circle. This was further developed in the fifth century by the Chinese mathematicians ZU CHONGZHI and ZU GENG to find the volume of a sphere. In the same century, the Indian mathematician ARYABHATA 
used a similar method in order to find the volume of a cube. More than 1000 years later, Johannes KEPLER invented the Kepler'sche Fassregel [1] (today also known as Simpson rule) in order to compute the (approximative) volume of (wine) barrels.

Based on the fundamental work of ISAAC NEWTON and GOTTFRIED WILHEM LEIBNIZ in the 18th century (see [2,3]), the first indubitable access to integration was given by BERNHARD RIEMANN in his Habilitation Thesis at the University of Göttingen [4]. The Riemann integral is the best known integral, taught in each Calculus course - an axiomatization of this functional, however, was given in the late 20th century only. We present here the approach given in [5, Theorem 1].

Theorem 1.1 Let $\varphi$ be continuous on an interval $[a, b]$, and let $I_{u}^{v}$ be defined for $a \leq u \leq v \leq b$. Suppose that

$$
I_{a}^{x+\Delta x}=I_{a}^{x}+I_{x}^{x+\Delta x}
$$

and

$$
\left(\min _{[x, x+\Delta x]} \varphi\right) \Delta x \leq I_{x}^{x+\Delta x} \leq\left(\max _{[x, x+\Delta x]} \varphi\right) \Delta x
$$

when $\Delta x>0$. Then

$$
I_{a}^{b}=\int_{a}^{b} \varphi(x) d x
$$

A rather big delay between construction and axiomatization can be observed also in the case of other well-known integrals such as the Choquet and the Sugeno integral. Possible reasons for that may be hidden in the following quotation of ALBERT EINSTEIN where he speaks about the modern development of a theory, using his own relativity theory as an example [6, pp. 238-239]:

"That is to say, the hypotheses from which one starts become ever more abstract and more remote from experience. But in return one comes closer to the preeminent goal of science, that of encompassing a maximum of empirical contents through logical deduction with a minimum of hypotheses or axioms."

We discuss a special class of integrals - $[0,1]$-valued discrete universal integrals - from an axiomatic point of view. After having recalled the basic notions of capacities (fuzzy measures) and [0,1]-valued discrete universal integrals, we shall present an axiomatic approach to the Lebesgue, Choquet and Sugeno integral. This will be followed by an axiomatic characterization of copula-based universal integrals. Finally, some recently introduced functionals are studied, including the induced Choquet integral and a Sugeno-type integral based on level dependent capacities.

\section{Capacities and $[0,1]$-Valued Discrete Universal Integrals}

Throughout this paper, we consider a fixed finite universe $X$, i.e., without loss of generality we may assume $X=\{1,2, \ldots, n\}$.

Definition 2.1 A capacity (or fuzzy measure) on $X$ is a non-decreasing set function $m: 2^{X} \rightarrow[0,1]$ which satisfies the boundary conditions $m(\emptyset)=0$ and $m(X)=1$. 
Requiring additional properties, one obtains special types of capacities: for example, additive capacities are just probability measures on $\left(X, 2^{X}\right)$, while maxitive capacities are possibility measures [7].

Let us denote by $\mathcal{M}_{n}$ the set of all capacities on $X$ and by $\mathcal{F}_{n}$ the set of all functions from $X$ to $[0,1]$. The concept of universal integrals, which can be defined for arbitrary capacities on arbitrary measurable spaces $(X, \mathcal{A})$ and for arbitrary measurable functions $f: X \rightarrow[0,1]$, was axiomatically introduced in [8].

In this contribution, we use $[0,1]$-valued discrete universal integrals as the general framework for our investigations.

Definition 2.2 A function $\mathbf{I}: \bigcup_{n \in \mathbb{N}}\left(\mathcal{M}_{n} \times \mathcal{F}_{n}\right) \rightarrow[0,1]$ is called a [0,1]-valued discrete universal integral if it satisfies the following axioms:

(A1) I is non-decreasing in each component;

(A2) $\mathbf{I}\left(m, \mathbf{1}_{E}\right)=m(E) \quad$ for all $X=\{1,2, \ldots, n\}, m \in \mathcal{M}_{n}$, and $E \subseteq X$;

(A3) $\mathbf{I}\left(m, c \cdot \mathbf{1}_{X}\right)=c \quad$ for all $X=\{1,2, \ldots, n\}, m \in \mathcal{M}_{n}$, and $c \in[0,1]$;

(A4) $\mathbf{I}\left(m_{1}, f_{1}\right)=\mathbf{I}\left(m_{2}, f_{2}\right) \quad$ for all pairs $\left(m_{1}, f_{1}\right) \in \mathcal{M}_{n_{1}} \times \mathcal{F}_{n_{1}}$ and $\left(m_{2}, f_{2}\right) \in \mathcal{M}_{n_{2}} \times \mathcal{F}_{n_{2}}$ satisfying $m_{1}\left(\left\{f_{1} \geq t\right\}\right)=m_{2}\left(\left\{f_{2} \geq t\right\}\right)$ for each $t \in[0,1]$.

It is not difficult to check that, as a consequence of (A4), the value $\mathbf{I}\left(m, c \cdot \mathbf{1}_{E}\right)$ depends on the constant $c \in[0,1]$ and the value $m(E) \in[0,1]$ only, i.e., there is an operation $\otimes:[0,1]^{2} \rightarrow[0,1]$ such that, for each $c \in[0,1]$ and each $E \subseteq\{1,2, \ldots, n\}$, we have $\mathbf{I}\left(m, c \cdot \mathbf{1}_{E}\right)=c \otimes m(E)$. This operation $\otimes$ turns out to be a semicopula [9], i.e., it is non-decreasing in each component and has 1 as neutral element:

Proposition 2.3 Let $\mathbf{I}$ be a $[0,1]$-valued discrete universal integral. Then there exists a semicopula $\otimes$ such that we have $\mathbf{I}\left(m, c \cdot \mathbf{1}_{E}\right)=c \otimes m(E)$ for all $n \in \mathbb{N}, m \in \mathcal{M}_{n}, c \in[0,1]$, and $E \subseteq\{1,2, \ldots, n\}$.

\section{Special $[0,1]$-Valued Discrete Universal Integrals}

Denote by $S_{n}$ the set of all permutations $\sigma:\{1,2, \ldots, n\} \rightarrow\{1,2, \ldots, n\}$. Given a capacity $m$ on a measurable space $(X, \mathcal{A})$ and a measurable function $f: X \rightarrow[0,1]$, the Choquet integral [10] is defined by

$$
\mathbf{C h}(m, f)=\int_{0}^{1} m(\{f \geq x\}) d x
$$

the integral on the right hand side being a classical Riemann integral. Considering a discrete universe $X=\{1,2, \ldots, n\}$, then for $(m, f) \in \mathcal{M}_{n} \times \mathcal{F}_{n}$ we obtain

$$
\mathbf{C h}(m, f)=\sum_{i=1}^{n} f(\sigma(i)) \cdot(m(\{\sigma(i), \ldots, \sigma(n)\})-m(\{\sigma(i+1), \ldots, \sigma(n)\}))
$$

where $\sigma \in S_{n}$ satisfies $f(\sigma(1)) \leq f(\sigma(2)) \leq \cdots \leq f(\sigma(n))$, and where the set $\{\sigma(n+1), \sigma(n)\}$ is defined to be the empty set $\emptyset$ as a convention.

Another way of looking at the discrete Choquet integral is using the so-called Lovász extension [11] (see also [12] who generalized the approach given in [13]):

$$
\mathbf{C h}(m, f)=\sum_{E \subseteq X} \mu(E) \cdot \min \{f(i) \mid i \in E\}
$$


where $X=\{1,2, \ldots, n\}$, and $\mu: 2^{X} \rightarrow \mathbb{R}$ is the Möbius transform of the capacity $m$ defined by

$$
\mu(E)=\sum_{F \subseteq E}(-1)^{\operatorname{card}(E \backslash F)} \cdot m(F)
$$

For more details about the discrete Choquet integral we recommend [14,15].

The axiomatization of the Choquet integral [16-18] is based on the notion of comonotonicity: two functions $f, g: X \rightarrow[0,1]$ are said to be comonotone (in symbols $f \sim g$ ) if, for all $i, j \in X$, we have $(f(i)-f(j)) \cdot(g(i)-g(j)) \geq 0$.

Theorem 3.1 Consider a function $\mathbf{J}: \bigcup_{n \in \mathbb{N}} \mathcal{F}_{n} \rightarrow[0,1]$. Then, for each $n \in \mathbb{N}$ there is a capacity $m \in \mathcal{M}_{n}$ such that for all $f \in \mathcal{F}_{n}$ we have $\mathbf{J}(f)=\mathbf{C h}(m, f)$ if and only if

(M) J is non-decreasing;

(C1) $\mathbf{J}\left(\mathbf{1}_{X}\right)=1 \quad$ for all $n \in \mathbb{N}$ and $X=\{1,2, \ldots, n\}$;

(C2) $\mathbf{J}$ is comonotone additive, i.e., for each $n \in \mathbb{N}$ and all $f, g \in \mathcal{F}_{n}$ satisfying $f+g \in \mathcal{F}_{n}$ and $f \sim g$ we have $\mathbf{J}(f+g)=\mathbf{J}(f)+\mathbf{J}(g)$.

Proof: The necessity is obvious. In order to show sufficiency, define first, for each $n \in \mathbb{N}$, the set function $m: 2^{\{1, \ldots, n\}} \rightarrow[0,1]$ by $m(E)=\mathbf{J}\left(\mathbf{1}_{E}\right)$ and show that $m \in \mathcal{M}_{n}$. Indeed, the boundary conditions of $m$ follow from $(\mathrm{C} 1)$ and $(\mathrm{C} 2)$, and $(\mathbf{M})$ implies that $m$ is non-decreasing. For $f \in \mathcal{F}_{n}$ and $\sigma \in S_{n}$ with $f(\sigma(1)) \leq f(\sigma(2)) \leq \cdots \leq f(\sigma(n))$ we have (using the convention $\sigma(0)=0$ )

$$
f=\sum_{i=1}^{n}(f(\sigma(i))-f(\sigma(i-1))) \cdot \mathbf{1}_{\{\sigma(i), \ldots, \sigma(n)\}}
$$

Because of $(\mathrm{C} 2)$ we obtain

$$
\mathbf{J}(f)=\sum_{i=1}^{n} \mathbf{J}\left((f(\sigma(i))-f(\sigma(i-1))) \cdot \mathbf{1}_{\{\sigma(i), \ldots, \sigma(n)\}}\right)
$$

Moreover, for each $E \subseteq\{1,2, \ldots, n\}$ and for each rational number $\left.\left.\frac{p}{q} \in\right] 0,1\right] \cap \mathbb{Q}$, property (C2) implies $\mathbf{J}\left(\frac{p}{q} \cdot \mathbf{1}_{E}\right)=\frac{p}{q} \cdot \mathbf{J}\left(\mathbf{1}_{E}\right)=\frac{p}{q} \cdot m(E)$. Then, because of $(\mathbf{M})$, we obtain $\mathbf{J}\left(c \cdot \mathbf{1}_{E}\right)=c \cdot m(E)$ for all $c \in[0,1]$ and $E \subseteq\{1,2, \ldots, n\}$, and the result follows.

For more details and alternative axiomatizations of the Choquet integral see [19].

Example 3.2 The requirement (M) in Theorem 3.1 cannot be omitted, as this counterexample shows: put $n=3$ and identify each function $f \in \mathcal{F}_{3}$ with the triplet $\left(x_{1}, x_{2}, x_{3}\right) \in[0,1]^{3}$, i.e., $f(i)=x_{i}$. Then the function $\mathbf{J}: \mathcal{F}_{3} \rightarrow[0,1]$ defined by

$$
\mathbf{J}\left(x_{1}, x_{2}, x_{3}\right)= \begin{cases}x_{1}+x_{2}+x_{3}-2 \cdot \operatorname{med}\left(x_{1}, x_{2}, x_{3}\right) & \text { if } x_{1} \vee x_{2} \leq x_{3} \\ x_{1} \vee x_{2} \vee x_{3} & \text { otherwise }\end{cases}
$$

has all the other properties required in Theorem 3.1 (for $n=3$ ). The corresponding set function $m$ is given by $m(\emptyset)=m(\{1,3\})=m(\{2,3\})=0$, and $m(E)=1$ otherwise. However, $m$ is not monotone and, therefore, not a capacity. 
The Sugeno integral [20] is defined by

$$
\mathbf{S u}(m, f)=\sup \{\min (x, m(\{f \geq x\})) \mid x \in[0,1]\}
$$

Its discrete form is

$$
\mathbf{S u}(m, f)=\sup \{\min (f(\sigma(i)), m(\{\sigma(i), \sigma(i+1), \ldots, \sigma(n)\})) \mid i \in X\}
$$

where $\sigma \in S_{n}$ satisfies $f(\sigma(1)) \leq f(\sigma(2)) \leq \ldots f(\sigma(n))$, or, equivalently (compare with Equation (3)),

$$
\mathbf{S u}(m, f)=\sup \{\min (m(E), \min \{f(i) \mid i \in E\}) \mid E \subseteq X\}
$$

An axiomatization of the Sugeno integral was given in [21] (compare also with [19]):

Theorem 3.3 Consider a function $\mathbf{J}: \bigcup_{n \in \mathbb{N}} \mathcal{F}_{n} \rightarrow[0,1]$. Then, for each $n \in \mathbb{N}$ there is a capacity $m \in \mathcal{M}_{n}$ such that for all $f \in \mathcal{F}_{n}$ we have $\mathbf{J}(f)=\mathbf{S u}(m, f)$ if and only if

(S1) $\mathbf{J}\left(\mathbf{1}_{X}\right)=1 \quad$ for all $n \in \mathbb{N}$ and $X=\{1,2, \ldots, n\}$;

(S2) $\mathbf{J}$ is comonotone maxitive, i.e., for each $n \in \mathbb{N}$ and all $f, g \in \mathcal{F}_{n}$ with $f \sim g$ we have $\mathbf{J}(\max (f, g))=\max (\mathbf{J}(f), \mathbf{J}(g))$;

(S3) $\mathbf{J}$ is min-homogeneous, i.e., for each $n \in \mathbb{N}$ and $X=\{1,2, \ldots, n\}$, for all $f \in \mathcal{F}_{n}$ and for all $c \in[0,1]$ we have $\mathbf{J}\left(\min \left(f, c \cdot \mathbf{1}_{X}\right)\right)=\min (\mathbf{J}(f), c)$.

Proof: The necessity is obvious. In order to show sufficiency, define, for each $n \in \mathbb{N}$, the set function $m: 2^{\{1, \ldots, n\}} \rightarrow[0,1]$ by $m(E)=\mathbf{J}\left(\mathbf{1}_{E}\right)$. Then (S1) and (S3) imply $m(X)=1$ and $\left.m(\emptyset)=0\right)$, and (S2) ensures that $m$ is non-decreasing, i.e., $m \in \mathcal{M}_{n}$. For the rest of the proof it is enough to take into account that each $f \in \mathcal{F}_{n}$ can be expressed in the form $f=\bigvee_{i=1}^{n} \min \left(f(\sigma(i)) \cdot \mathbf{1}_{X}, \mathbf{1}_{\{\sigma(i), \ldots, \sigma(n)\}}\right)$.

Adding also the axiom of symmetry, i.e.,

(SY) $\mathbf{J}(f)=\mathbf{J}(f \circ \sigma) \quad$ for all $n \in \mathbb{N}, f \in \mathcal{F}_{n}$ and $\sigma \in S_{n}$

we obtain an axiomatic characterization of both the ordered weighted averaging operators (briefly OWA operators) [22] and the ordered weighted maximum (OWMax operators for short) [23]:

Corollary 3.4 A function $\mathbf{J}: \bigcup_{n \in \mathbb{N}} \mathcal{F}_{n} \rightarrow[0,1]$ is

(i) an OWA operator if and only if it satisfies the axioms (C1), (C2), (M) and (SY);

(ii) an OWMax operator if and only if it satisfies the axioms (S1), (S2), (S3) and (SY).

On the other hand, stronger versions of some of these axioms lead to special subclasses of the discrete integrals discussed above:

Corollary 3.5 Consider a function $\mathbf{J}: \bigcup_{n \in \mathbb{N}} \mathcal{F}_{n} \rightarrow[0,1]$. Then, for each $n \in \mathbb{N}$ there is a probability measure $m \in \mathcal{M}_{n}$ such that for all $f \in \mathcal{F}_{n}$ we have

$$
\mathbf{J}(f)=\int_{\{1, \ldots, n\}} f d m
$$

if and only if $\mathbf{J}$ satisfies the axioms $(\mathrm{C} 1)$ and 
$\left(\mathrm{C} 2^{*}\right) \mathrm{J}$ is additive.

Observe that in Corollary 3.5 the monotonicity of $\mathbf{J}$ follows from the additivity $\left(\mathrm{C} 2^{*}\right)$ and the fact that $\operatorname{Ran}(\mathbf{J}) \subseteq[0,1]$

Corollary 3.6 Suppose that a function $\mathbf{J}: \bigcup_{n \in \mathbb{N}} \mathcal{F}_{n} \rightarrow[0,1]$ satisfies the axioms (S1), (S3) and $\left(\mathrm{S} 2^{*}\right) \mathbf{J}$ is maxitive.

Then, for each $n \in \mathbb{N}$, J is the weighted maximum on $\mathcal{F}_{n}$, i.e., for each $f \in \mathcal{F}_{n}$ we have

$$
\mathbf{J}(f)=\max \left\{\min \left(w_{i, n}, f(i)\right) \mid i \in\{1,2, \ldots, n\}\right\}
$$

where, for each $i \in\{1,2, \ldots, n\}, w_{i, n}=\mathbf{J}\left(f_{i, n}\right)$ and $f_{i, n}=\mathbf{1}_{\{i\}} \in \mathcal{F}_{n}$.

Observe that, for a given semicopula $\otimes$, the smallest discrete universal integral I satisfying, for each $n \in \mathbb{N}$, each $m \in \mathcal{M}_{n}$, each $E \subseteq\{1, \ldots, n\}$ and each $c \in[0,1]$, the equality $\mathbf{I}\left(m, c \cdot \mathbf{1}_{E}\right)=c \otimes m(E)$ is denoted by $\mathbf{I}_{\otimes}$ (see [8]). The explicit formula of $\mathbf{I}_{\otimes}$ is

$$
\mathbf{I}_{\otimes}(m, f)=\sup \{f(\sigma(i)) \otimes m(\{\sigma(i), \ldots, \sigma(n)\}) \mid i \in\{1, \ldots, n\}\}
$$

where the permutation $\sigma \in S_{n}$ has the same properties as the permutations appearing in Equations (2) and (6).

Then the Sugeno integral corresponds to the greatest semicopula $\wedge$, given by $x \wedge y=\min (x, y)$, i.e., $\mathbf{I}_{\wedge}=\mathbf{S u}$. Moreover, for the product semicopula $\Pi$, given by $\Pi(x, y)=x \cdot y$, we obtain the Shilkret integral [40]. As a consequence, Theorem 3.3 can be strengthened and generalized as follows:

Theorem 3.7 Let $\otimes$ be a semicopula and consider a function $\mathbf{J}: \bigcup_{n \in \mathbb{N}} \mathcal{F}_{n} \rightarrow[0,1]$. Then, for each $n \in \mathbb{N}$ there is a capacity $m \in \mathcal{M}_{n}$ such that for each $f \in \mathcal{F}_{n}$ we have $\mathbf{J}(f)=\mathbf{I}_{\otimes}(m, f)$ if and only if $\mathbf{J}$ satisfies the axioms (S1), (S2) and

$\left(\mathrm{S} 3_{\otimes}\right) \mathbf{J}$ is $\otimes$-homogeneous on characteristic functions, i.e., for each $n \in \mathbb{N}$, for all $E \subseteq\{1, \ldots, n\}$ and for all $c \in[0,1]$ we have $\mathbf{J}\left(c \otimes \mathbf{1}_{E}\right)=c \otimes \mathbf{J}\left(\mathbf{1}_{E}\right)$.

Proof: The necessity is obvious. In order to show the sufficiency, we can proceed in the same way as in the proof of Theorem 3.3, taking into account that for each $n \in \mathbb{N}$ and each $f \in \mathcal{F}_{n}$ we have

$$
f=\max _{i \in\{1, \ldots, n\}} f\left(\sigma(i) \otimes \mathbf{1}_{\{\sigma(i), \ldots, \sigma(n)\}}\right)
$$

the permutation $\sigma \in S_{n}$ again having the same properties as in Equations (2) and (6), and the fact that the system of functions $\left(f\left(\sigma(i) \otimes \mathbf{1}_{\{\sigma(i), \ldots, \sigma(n)\}}\right)_{i \in\{1, \ldots, n\}}\right.$ is a comonotone system.

Remark 3.8 As a consequence of Theorem 3.7, the axiom (S3) of min-homegeneity in the axiomatic characterization of the Sugeno integral in Theorem 3.3 can be replaced by the weaker axiom $\left(\mathrm{S} 3_{\wedge}\right)$, requiring the min-homogeneity for characteristic functions only. 


\section{Copula-Based [0, 1]-Valued Discrete Universal Integrals}

Copulas were introduced in [24] to model the dependence structure of random vectors (for a detailed treatise see [25]). Here we restrict ourselves to two-dimensional copulas only which also can be considered as special binary aggregation functions or, more precisely, as special semicopulas.

Definition 4.1 A function $C:[0,1]^{2} \rightarrow[0,1]$ is called a (2-dimensional) copula if it is a 2-increasing semicopula, i.e., if for all $x, y, x^{*}, y^{*} \in[0,1]$ with $x \leq x^{*}$ and $y \leq y^{*}$ we have

$$
C\left(x^{*}, y^{*}\right)-C\left(x^{*}, y\right)-C\left(x, y^{*}\right)+C(x, y) \geq 0
$$

Note that copulas are in a one-to-one correspondence with probability measures on the Borel subsets $\mathcal{B}\left([0,1]^{2}\right)$ with uniform marginals, i.e., for each copula $C:[0,1]^{2} \rightarrow[0,1]$ there is a unique probability measure $P_{C}: \mathcal{B}\left([0,1]^{2}\right) \rightarrow[0,1]$ satisfying $P_{C}([0, a] \times[0, b])=C(a, b)$. Based on ideas in [26], in [27] (see also [8]), for a given copula $C$ the copula-based integral $\mathbf{I}_{C}$ was introduced as

$$
\mathbf{I}_{C}(m, f)=P_{C}\left(\left\{(x, y) \in[0,1]^{2} \mid y \leq m(\{f \geq x\})\right\}\right)
$$

As already mentioned in the previous section, for the independence copula $\Pi$ we rediscover the Choquet integral, i.e., $\mathbf{I}_{\Pi}=\mathbf{C h}$, whereas for the greatest copula $\wedge$ we obtain the Sugeno integral, i.e., $\mathbf{I}_{\wedge}=\mathbf{S u}$.

In the discrete case we get (again $\sigma \in S_{n}$ has the same properties as in Equations (2) and (6))

$$
\mathbf{I}_{C}(m, f)=\sum_{i=1}^{n}(C(f(\sigma(i)), m(\{\sigma(i), \ldots, \sigma(n)\}))-C(f(\sigma(i)), m(\{\sigma(i+1), \ldots, \sigma(n)\})))
$$

In [28], we recently gave an axiomatization of copula-based [0, 1]-valued discrete integrals for a fixed $n \in \mathbb{N}$. We modify the main result here for arbitrary $n \in \mathbb{N}$ :

Theorem 4.2 Consider a function $\mathbf{J}: \bigcup_{n \in \mathbb{N}} \mathcal{F}_{n} \rightarrow[0,1]$. Then, for each $n \in \mathbb{N}$ there is a capacity $m \in \mathcal{M}_{n}$ and a copula $C$ such that for all $f \in \mathcal{F}_{n}$ we have $\mathbf{J}(f)=\mathbf{I}_{C}(m, f)$ if and only if $\mathbf{J}$ satisfies the axioms $(\mathrm{M})$ and

(K1) $\mathbf{J}\left(c \cdot \mathbf{1}_{X}\right)=c \quad$ for all $n \in \mathbb{N}, X=\{1,2, \ldots, n\}$ and $c \in[0,1]$;

(K2) $\mathbf{J}$ is comonotone modular, i.e., for each $n \in \mathbb{N}$ and for all $f, g \in \mathcal{F}_{n}$ with $f \sim g$ we have

$$
\mathbf{J}(\max (f, g))+\mathbf{J}(\min (f, g))=\mathbf{J}(f)+\mathbf{J}(g)
$$

(K3) for all $n \in \mathbb{N}, E, F \subseteq\{1,2, \ldots, n\}$ and $(u, v) \in[0,1]^{2}$ we have

(i) if $\mathbf{J}\left(\mathbf{1}_{E}\right)=\mathbf{J}\left(\mathbf{1}_{F}\right)$ then $\mathbf{J}\left(u \cdot \mathbf{1}_{E}\right)=\mathbf{J}\left(u \cdot \mathbf{1}_{F}\right)$;

(ii) if $u \leq v$ and $\mathbf{J}\left(\mathbf{1}_{E}\right) \leq \mathbf{J}\left(\mathbf{1}_{F}\right)$ then $\mathbf{J}\left(u \cdot \mathbf{1}_{F}\right)-\mathbf{J}\left(u \cdot \mathbf{1}_{E}\right) \leq \mathbf{J}\left(v \cdot \mathbf{1}_{F}\right)-\mathbf{J}\left(v \cdot \mathbf{1}_{E}\right)$.

Proof: The necessity is obvious. In order to show sufficiency, define, for each $n \in \mathbb{N}$, the capacity $m \in$ $\mathcal{M}_{n}$ by $m(E)=\mathbf{J}\left(\mathbf{1}_{E}\right)$, and the copula $C$ as an extension of the function $D:[0,1] \times \operatorname{Ran}(m) \rightarrow[0,1]$ given by $D(u, m(E))=\mathbf{J}\left(u \cdot \mathbf{1}_{E}\right)$ for each $E \subseteq\{1,2, \ldots, n\}$. For the rest of the proof see [28].

In [29] the so-called ordered modular average (OMA operator for short) was introduced and studied: given a system $\Phi=\left\{\varphi_{i, n}:[0,1] \rightarrow[0,1] \mid n \in \mathbb{N}, i \in\{1,2, \ldots, n\}\right\}$ of non-decreasing real functions satisfying $\sum_{i=1}^{n} \varphi_{i, n}=\operatorname{id}_{[0,1]}$, then the function

$$
\mathrm{OMA}_{\Phi}: \bigcup_{n \in \mathbb{N}} \mathcal{F}_{n} \rightarrow[0,1]
$$


given, for $f \in \mathcal{F}_{n}$, by

$$
\mathrm{OMA}_{\Phi}(f)=\sum_{i=1}^{n} \varphi_{i, n}(f(\sigma(i)))
$$

is called an OMA operator. Note that in the case $\varphi_{i, n}=w_{i, n} \cdot \mathrm{id}_{[0,1]}$, the OWA and the OMA operator coincide.

Corollary 4.3 A function $\mathbf{J}: \bigcup_{n \in \mathbb{N}} \mathcal{F}_{n} \rightarrow[0,1]$ is an OMA operator if and only if it satisfies the axioms (K1), (K2) and (SY).

\section{Generalizations of Choquet and Sugeno Integrals}

During the last years, several new types of integrals were considered which generalize or modify those discussed in the previous sections. For example, Choquet and Sugeno type integrals based on level dependent capacities were studied in [30], [31] and [32]. Recall that, for $X=\{1,2, \ldots, n\}$, a function $M: 2^{X} \times[0,1] \rightarrow[0,1]$ is called a level dependent capacity on $X$ if, for each $t \in[0,1], m_{t}(\cdot)=M(\cdot, t)$ is a capacity on $X$, i.e., $M=\left(m_{t}\right)_{t \in[0,1]}$.

The Sugeno integral with respect to a level dependent capacity $M=\left(m_{t}\right)_{t \in[0,1]}$ is then given by

$$
\mathbf{S u}(M, f)=\sup \left\{\min \left(t, m_{t}(\{f \geq t\}) \mid t \in[0,1]\right\}\right.
$$

This is an extension of the Sugeno integral in the sense that for a constant level dependent capacity $M=\left(m_{t}\right)_{t \in[0,1]}$, i.e., if there is a capacity $m$ such that $m_{t}=m$ for each $t \in[0,1]$, we obtain the Sugeno integral with respect to $m$, i.e., $\mathbf{S u}(M, \cdot)=\mathbf{S u}(m, \cdot)$.

Based on results in [32] and [33, Proposition 2.127], we can derive the following axiomatization of the Sugeno integral based on level dependent capacities:

Theorem 5.1 Consider a function $\mathbf{J}: \bigcup_{n \in \mathbb{N}} \mathcal{F}_{n} \rightarrow[0,1]$. Then, for each $n \in \mathbb{N}$ there is a level dependent capacity $M=\left(m_{t}\right)_{t \in[0,1]}$ with $m_{t} \in \mathcal{M}_{n}$ for all $t \in[0,1]$, such that for all $f \in \mathcal{F}_{n}$ we have $\mathbf{J}(f)=\mathbf{S u}(M, f)$ if and only if $\mathbf{J}$ satisfies the axioms (K1) and (S2).

Note that the smallest level dependent capacity $M_{*}: 2^{\{1, \ldots, n\}} \times[0,1] \rightarrow[0,1]$ that is linked to a function $\mathbf{J}: \bigcup_{n \in \mathbb{N}} \mathcal{F}_{n} \rightarrow[0,1]$ such that (as in Theorem 5.1) $\mathbf{J}(f)=\mathbf{S u}\left(M_{*}, f\right)$ for all $n \in \mathbb{N}$ and for all $f \in \mathcal{F}_{n}$ is given by

$$
M_{*}(E, t)= \begin{cases}1 & \text { if } E=\{1,2, \ldots, n\}, \\ \mathbf{J}\left(t \cdot \mathbf{1}_{E}\right) & \text { otherwise. }\end{cases}
$$

A modification of the Choquet integral based on so-called order inducing variables was proposed in [34]. The induced Choquet integral on $X=\{1,2, \ldots, n\}$ based on a capacity $m \in \mathcal{M}_{n}$ and order inducing variables $\left(u_{1}, u_{2}, \ldots, u_{n}\right) \in[0,1]^{n}$ is defined by

$$
\begin{aligned}
\mathbf{I C h}\left(m,\left(u_{1}, \ldots, u_{n}\right), f\right)= & \mathbf{A M}\left\{\sum_{i=1}^{n} f(\sigma(i)) \cdot(m(\{\sigma(i), \ldots, \sigma(n)\})\right. \\
& \left.-m(\{\sigma(i+1), \ldots, \sigma(n)\})) \mid \sigma \in S_{n} \text { with } u_{\sigma(1)} \leq u_{\sigma(2)} \leq \cdots \leq u_{\sigma(n)}\right\}
\end{aligned}
$$


where $\mathbf{A M}$ is the arithmetic mean and the set $\{\sigma(n+1), \sigma(n)\}$ is defined to be the empty set $\emptyset$ as a convention.

The axiomatization of the induced Choquet integral was given in [35].

Theorem 5.2 Fix $n \in \mathbb{N}$ and consider a function $\mathbf{J}: \bigcup_{n \in \mathbb{N}} \mathcal{F}_{n} \rightarrow[0,1]$. Then there is a capacity $m \in$ $\mathcal{M}_{n}$ such that for all $f \in \mathcal{F}_{n}$ and for all $\mathbf{u}=\left(u_{1}, u_{2}, \ldots, u_{n}\right) \in[0,1]^{n}$ we have $\mathbf{J}(\mathbf{u}, f)=\mathbf{I C h}(m, \mathbf{u}, f)$ if and only if $\mathbf{J}$ satisfies the axioms

(IC1) $\mathbf{J}\left(\mathbf{u}, \mathbf{1}_{\{1,2, \ldots, n\}}\right)=1 \quad$ for each $\mathbf{u} \in[0,1]^{n}$;

(IC2) $\mathbf{J}(\mathbf{u}, \cdot)$ is additive for each $\mathbf{u} \in[0,1]^{n}$;

(IC3) $\mathbf{J}\left(\mathbf{u}, \mathbf{1}_{E}\right)=\mathbf{J}\left(\mathbf{v}, \mathbf{1}_{E}\right) \quad$ for all $E \subseteq\{1,2, \ldots, n\}$ and for all $\mathbf{u}, \mathbf{v} \in[0,1]^{n}$ satisfying $u_{i}>u_{j}$ and $v_{i}>v_{j}$ whenever $i \in E$ and $j \notin E$;

(IC4) $\mathbf{J}(\mathbf{u}, \cdot)=\mathbf{A M}\left\{\mathbf{J}\left(\left(\frac{\sigma(1)}{n}, \frac{\sigma(2)}{n}, \ldots, \frac{\sigma(n)}{n}\right), \cdot\right) \mid \sigma \in S_{n},(\sigma(1), \sigma(2), \ldots, \sigma(n)) \sim \mathbf{u}\right\}$.

Proof: The necessity is obvious. In order to show sufficiency, define the set function $m \in \mathcal{M}_{n}$ by $m(E)=\mathbf{J}\left(\mathbf{v}, \mathbf{1}_{E}\right)$, where $\mathbf{v} \in[0,1]^{n}$ satisfies $v_{i}>v_{j}$ whenever $i \in E$ and $j \notin E$. Note that $m$ is well-defined because of (IC3), and that (IC1) and (IC2) imply $m(X)=1$ and the monotonicity of $m$. For the rest of the proof see [35].

\section{Concluding Remarks}

Axiomatization, as observed in [6], of a theory, a model or a construction is a highly condensed piece of information which covers a big number of empirical facts. We have presented axiomatic approaches to different types of integrals, restricting ourselves to finite domains and ranges in the unit interval. Several of the results presented here are well-known from the literature, especially those related to the Choquet and the Sugeno integral. However, we also give new results (e.g., Theorem 3.7) and some recent results (most of them in Section 5) which have been discovered during the past two years.

Obviously, $[0,1]$ can be extended to $[0, \infty]$ or $[-\infty, \infty]$, and/or an abstract measurable space $(X, \mathcal{A})$ can be considered - usually this means that the axioms have to be modified.

As an example, consider the Choquet integral: if $(X, \mathcal{A})$ is a measurable space, if $\mathcal{F}$ denotes the set of all measurable functions $f: X \rightarrow[0,1]$, and if $\mathbf{J}: \mathcal{F} \rightarrow[0,1]$ is a non-decreasing function, then there exists a capacity $m: \mathcal{A} \rightarrow[0,1]$ such that for all $f \in \mathcal{F}$ we have

$$
\mathbf{J}(f)=\mathbf{C h}(m, f)=\int_{0}^{\infty} m(\{f \geq x\}) d x
$$

if and only if $\mathbf{J}\left(\mathbf{1}_{X}\right)=1$ and if $\mathbf{J}$ is comonotone additive [16,17] (compare with Theorem 3.1).

Applying these axioms to functions $f: X \rightarrow[-\infty, \infty]$, one obtains the asymmetric Choquet integral [36]

$$
\mathbf{C h}_{\mathrm{AS}}(m, f)=\int_{0}^{\infty} m(\{f \geq x\}) d x-\int_{-\infty}^{0}(1-m(\{f \geq x\})) d x=\mathbf{C h}\left(m, f^{+}\right)-\mathbf{C h}\left(m^{d}, f^{-}\right)
$$

where $f^{+}=\max (f, 0), f^{-}=\max (-f, 0)$, and $m^{d}: \mathcal{A} \rightarrow[0,1]$ is given by $m^{d}(E)=1-m(X \backslash E)$. 
Requiring the comonotone additivity for non-negative functions only, and adding a symmetry property, i.e., $\mathbf{J}(-f)=-\mathbf{J}(f)$, one obtains the symmetric Choquet integral (or Šipoš integral) [37]

$$
\mathbf{C h}_{\mathbf{S}}(m, f)=\mathbf{C h}\left(m, f^{+}\right)-\mathbf{C h}\left(m, f^{-}\right)
$$

Similar considerations for the Sugeno integral can be found in [38].

On the other hand, there are integrals based on some generalization of capacities. As an example, we mention the axiomatization of a Choquet type integral based on a signed capacity given in [39].

Finally, let us mention that several types of integrals have been introduced whose axiomatizations were not yet studied. Some of them are related to subsystems of the power set of a given finite universe. This is the case for the original Šipoš integral [37], the integral proposed in [41] or the subdecompositionbased integral studied in [42]. Similarly, hierarchical approaches to integrals, e.g., two-step Choquet integrals [43,44], as well as mixtures of integrals [45-47] were not yet axiomatized.

\section{Acknowledgment}

The second author was supported by the following grants: APVV-0073-10 and VEGA 1/0171/12. The authors would like to thank the two anonymous referees whose comments led to improvements of the original manuscript.

\section{References}

1. Kepler, J. Nova Stereometria Doliorum Vinariorum; 1615.

2. Leibniz, G.W. Nova Methodus Pro Maximis et Minimis; 1684.

3. Newton, I. Philosophice Naturalis Principia Mathematica; 1687.

4. Riemann, B. Über die Darstellbarkeit Einer Function Durch Eine Trigonometrische Reihe. Habilitation Thesis, University of Göttingen: Göttingen, Germany, 1854.

5. Gillman, L. An axiomatic approach to the integral. Am. Math. Mon. 1993, 100, 16-25.

6. Einstein, A. Ideas and Opinions; Bonanza Books: New York, NY, USA, 1954.

7. Zadeh, L.A. Fuzzy sets as a basis for a theory of possibility. Fuzzy Sets Syst. 1978, 1, 3-28.

8. Klement, E.P.; Mesiar, R.; Pap, E. A universal integral as common frame for Choquet and Sugeno integral. IEEE Trans. Fuzzy Syst. 2010, 18, 178-187.

9. Bassan, B.; Spizzichino, F. Relations among univariate aging, bivariate aging and dependence for exchangeable lifetimes. J. Multivar. Anal. 2005, 93, 313-339.

10. Choquet, G. Theory of capacities. Ann. Inst. Fourier (Grenoble) 1954, 5, 131-295.

11. Lovász, L. Submodular Functions and Convexity. In Mathematical Programming: The State of the Art (Bonn, 1982); Springer: Berlin, Germany, 1983; pp. 235-257.

12. Chateauneuf, A.; Jaffray, J.Y. Some characterizations of lower probabilities and other monotone capacities through the use of Möbius inversion. Math. Soc. Sci. 1989, 17, 263-283.

13. Dempster, A.P. Upper and lower probabilities induced by a multivalued mapping. Ann. Math. Stat. 1967, 38, 325-339.

14. Grabisch, M. Fuzzy integral in multicriteria decision making. Fuzzy Sets Syst. 1995, 69, 279-298. 
15. Grabisch, M. Set Functions Over Finite Sets: Transformations and Integrals. Handbook of Measure Theory; Pap, E., Ed.; Elsevier Science: Amsterdam, The Netherlands, 2002; Chapter 34, pp. 1381-1401.

16. Schmeidler, D. Integral representation without additivity. Proc. Am. Math. Soc. 1986, 97, 255-261.

17. Schmeidler, D. Subjective probability and expected utility without additivity. Econometrica 1989, 57, 571-587.

18. Marichal, J.L. An axiomatic approach of the discrete Choquet integral as a tool to aggregate interacting criteria. IEEE Trans. Fuzzy Syst. 2000, 8, 800-807.

19. Benvenuti, P.; Mesiar, R.; Vivona, D. Monotone Set Functions-Based Integrals. Handbook of Measure Theory; Pap, E., Ed.; Elsevier Science: Amsterdam, The Netherlands, 2002; Chapter 33, pp. 1329-1379.

20. Sugeno, M. Theory of Fuzzy Integrals and Its Applications. Ph.D. Thesis, Tokyo Institute of Technology, Tokyo, Japan, 1974.

21. Marichal, J.L. An axiomatic approach of the discrete Sugeno integral as a tool to aggregate interacting criteria in a qualitative framework. IEEE Trans. Fuzzy Syst. 2001, 9, 164-172.

22. Yager, R.R. On ordered weighted averaging aggregation operators in multicriteria decisionmaking. IEEE Trans. Syst. Man Cybern. 1988, 18, 183-190.

23. Dubois, D.; Prade, H. Fuzzy sets in approximate reasoning-Part 1: Inference with possibility distributions. Fuzzy Sets Syst. 1991, 40, 143-202.

24. Sklar, A. Fonctions de répartition à $n$ dimensions et leurs marges. Publ. Inst. Stat. Univ. Paris 1959, 8, 229-231.

25. Nelsen, R.B. An Introduction to Copulas, 2nd ed.; Springer: New York, NY, USA, 2006.

26. Imaoka, H. On a subjective evaluation model by a generalized fuzzy integral. Int. J. Uncertain. Fuzz. Knowl.-B. 1997, 5, 517-529.

27. Klement, E.P.; Mesiar, R.; Pap, E. Measure-based aggregation operators. Fuzzy Sets Syst. 2004, 142, 3-14.

28. Klement, E.P.; Mesiar, R. On the axiomatization of some classes of discrete universal integrals. Knowl.-Based Syst. 2012, in press.

29. Mesiar, R.; Mesiarová-Zemánková, A. The ordered modular averages. IEEE Trans. Fuzzy Syst. 2011, 19, 42-50.

30. Giove, S.; Greco, S.; Matarazzo, B. Level Dependent Capacities and the Generalized Choquet Integral. In Abstracts of the 28th Linz Seminar on Fuzzy Set Theory: "Fuzzy Sets, Probability, and Statistics—Gaps and Bridges”; Dubois, D., Klement, E.P., Mesiar, R., Eds.; Johannes Kepler University: Linz, Austria, 2007; pp. 54-55.

31. Greco, S.; Matarazzo, B.; Giove, S. The Choquet integral with respect to a level dependent capacity. Fuzzy Sets Syst. 2011, 175, 1-35.

32. Mesiar, R.; Mesiarová-Zemánková, A.; Ahmad, K. Level-dependent Sugeno integral. IEEE Trans. Fuzzy Syst. 2009, 17, 167-172.

33. Grabisch, M.; Marichal, J.L.; Mesiar, R.; Pap, E. Aggregation Functions; Cambridge University Press: Cambridge, UK, 2009. 
34. Yager, R.R. Choquet aggregation using order inducing variables. Int. J. Uncertain. Fuzz. Knowl.B. 2004, 12, 69-88.

35. Greco, S.; Mesiar, R. Induced Choquet Integrals. IIn Proceedings of the IPMU 2012, (to appear).

36. Denneberg, D. Non-additive Measure and Integral; Kluwer Academic Publishers: Dordrecht, The Netherlands, 1994.

37. Šipoš, J. Integral with respect to a pre-measure. Math. Slovaca 1979, 29, 141-155.

38. Grabisch, M. The symmetric Sugeno integral. Fuzzy Sets Syst. 2003, 139, 473-490.

39. Cardin, M.; Couceiro, M.; Giove, S.; Marichal, J.L. Axiomatizations of signed discrete Choquet integrals. Int. J. Uncertain. Fuzz. Knowl.-B. 2011, 19, 193-199.

40. Shilkret, N. Maxitive measure and integration. Indag. Math. 1971, 33, 109-116.

41. Faigle, U.; Grabisch, M. A discrete Choquet integral for ordered systems. Fuzzy Sets Syst. 2011, $168,3-17$.

42. Eveny, Y.; Lehrer, E. Decomposition-Integral: Unifying Choquet and the Concave Integrals; Tel Aviv University: Tel Aviv, Israel (preprint), 2011.

43. Sugeno, M.; Fujimoto, K.; Murofushi, K. Hierarchical decomposition of Choquet integral models. Int. J. Uncertain. Fuzz. Knowl.-B. 1995, 3, 1-15.

44. Mesiar, R.; Vivona, D. Two-step integral with respect to fuzzy measure. Tatra Mt. Math. Publ. 1999, 16, 359-368.

45. Torra, V. Twofold integral: A Choquet integral and Sugeno integral generalization [in Catalan]. Bull. Assoc. Catal. Intell. Artif. 2003, 29, 14-20.

46. Narukawa, Y.; Torra, V. Twofold integral and multi-step Choquet integral. Kybernetika (Prague) 2004, 40, 39-50.

47. Narukawa, Y; Torra, V. Generalized transformed t-conorm integral and multifold integral. Fuzzy Sets Syst. 2006, 157, 1384-1392.

(c) 2012 by the authors; licensee MDPI, Basel, Switzerland. This article is an open access article distributed under the terms and conditions of the Creative Commons Attribution license (http://creativecommons.org/licenses/by/3.0/). 